\title{
Comparing Ultrasound-Guided Fine Needle Aspiration Techniques for Thyroid Nodules - Is The Short Axis Better Than The Long Axis?
}

Raed Farhat ( $\nabla$ raed.frhat88@gmail.com )

Ziv Medical Center

Leemor Wallach

Ziv Medical Center

Yaniv Avraham

Ziv Medical Center

Alex Tsipis

Ziv Medical center

Adi Sharabi-Nov

Ziv Medical Center

Nidal Alkhatib

Ziv Medical Center

Shlomo Merchavy

Ziv Medical Center

\section{Research Article}

Keywords: Ultrasound-guided fine needle aspiration cytology, thyroid nodule, long axis technique, short axis technique

Posted Date: October 15th, 2021

DOI: https://doi.org/10.21203/rs.3.rs-943069/v1

License: (c) (i) This work is licensed under a Creative Commons Attribution 4.0 International License. Read Full License 


\section{Abstract}

Background: Ultrasound-guided fine needle aspiration cytology (FNAC US) has been proven to be an accurate and efficient tool in thyroid nodule evaluation. Thyroid nodule aspiration can be acquired with either of two techniques: the short axis, in which only the tip of the needle is observed, and the long axis, in which the entire length of the needle is observed. Our retrospective study aimed to compare the adequacy of the two techniques.

Methods: FNAC US was performed in 357 thyroid nodules between January 2019 and December 2019. Data on the technique and the diagnostic accuracy were collected.

Results: A total of 181 nodules were aspirated using the long axis technique, and 176 nodules were aspirated using the short axis technique. The diagnostic adequacies of the long axis technique were significantly higher than those of the short axis technique. technique ( $93 \%$ versus $83 \%$, respectively, $p<$ $0.001)$.

Conclusion: In our study, the long axis technique provided more accurate cytological evaluation than the short axis technique.

\section{Introduction}

Thyroid nodules are very common and are diagnosed in $34 \%$ (27\% in men, $41.7 \%$ in women) of the adult population. The majority of nodules are benign, while $5 \%-15 \%$ of those nodules are malignant. Ultrasonography (US) is the most important diagnostic tool in the workup of thyroid nodules.

The clinical importance of thyroid nodule diagnosis rests with the need to exclude thyroid cancer, which occurs in $7 \%-15 \%$ of cases with correspondence to age, sex, previous exposure to ionizing radiation, family history, and other factors. ${ }^{3}$

The most acceptable US classification for thyroid nodules is the American College of Radiology Thyroid Imaging, Reporting and Data System (ACR TI-RADS). The score is based on the shape, echogenicity, composition, and margins of the nodule.

In the last decade, the US-guided fine needle aspiration cytology (FNAC) diagnostic method has been the gold standard for cytopathology differentiation between benign and malignant nodules. US-guided FNAC has been shown to have high rates of sensitivity and specificity, as well as diagnostic accuracy. The Bethesda system for reporting thyroid cytopathology (TBSRTC) was set up in 2007 by the National Cancer Institute, which standardized the reporting of thyroid cytopathology. The six diagnostic categories defined in this system are linked to certain ranges of malignancy risk and clinical management guidelines.

In TBSRTC, inadequate samples are pathologically reported as Category I. This category applies to samples that are nondiagnostic or unsatisfactory due to obscuring blood, overly thick smears, air drying 
of alcohol-fixed smears, or an inadequate number of follicular cells.

Despite the established efficacy of US-guided FNAC in the workup of thyroid masses, relatively high nondiagnostic (Category I) rates continue to hinder the full potential of FNAC. These rates for ultrasoundguided FNAC in the literature range widely from 2 to $29 \%$. Such nondiagnostic results increase costs, patient stress, and time to diagnosis. Most importantly, studies have shown that initial nondiagnostic samples can harbor a malignancy in $1-4 \%$ of cases.

The US-guided FNAC procedure can be performed using either the short axis (perpendicular) or a long axis (parallel) approach to visualize the needle as it is advanced toward the desired nodule. In a short axis approach, the ultrasound transducer is oriented transversely to the thyroid nodule. When the needle is inserted perpendicular to the transducer, it bisects the plain of imaging and therefore appears as a hyperechoic "dot" on the ultrasound screen (Fig. 1A-B/1A-C). In a long axis approach, the needle is inserted parallel to the ultrasound transducer's longest axis under the center of the transducer and therefore lies completely within the plain of the imaging (Fig. 2A-B/Fig. 2A-C).

We hypothesized that US-guided FNAC performed on the long axis will produce more accurate results since the needle can be visualized throughout its course. The purpose of this study was to compare the long- and short-axis approaches and the rate of nondiagnostic FNAC results (Category I).

\section{Materials And Methods}

\subsection{Patients}

A retrospective study review was performed on 357 patient files with thyroid nodules who underwent US FNAC in our institution between January 2019 and December 2019.

Medical records, ultrasound findings, and cytologic results in all patients were retrospectively reviewed.

Thyroid nodule evaluation using ACR TI-RADS was performed prior to FNAC by the attending specialist.

All methods were carried out in accordance with relevant guidelines and regulations.

All experimental protocols were approved by the Ziv Medical Center human ethics Helsinki committee. Informed consent was obtained from all subjects and/or their legal guardian(s).

\subsection{US-Guided FNAC Procedure.}

US-guided FNAC was performed by two expert physicians with more than 5 years FNAC US guided experience. As a routine, one expert uses the short axis approach, while the other uses the long axis approach. Patients are referred to either expert according to on-site availability.

All FNACs were performed using a 5-12 MHz linear transducer. 


\subsection{Cytologic preparation and diagnosis.}

The collected material was expelled on glass slides, smeared and fixed in $95 \%$ ethyl alcohol immediately following FNAC. Staining was performed using the Papanicolaou method.

An adequate sample was defined as having at least six clusters of thyroid follicular cells (15-20 cells) for each specimen.

All the samples were reported using the Bethesda System as follows :nondiagnostic (category I), (ND); (II) benign; (III) atypia or follicular lesion of undetermined significance (AUS/FLUS); (IV) follicular neoplasm or suspicious for follicular neoplasm (FN/SFN); (V) suspicious for malignancy (SM); and (VI) malignant.67

The rate of nondiagnosis (category I) between the two techniques mentioned above were compared, as well as other categories of the samples that were obtained.All slides were reviewed first by the attending cytopathologist, and subsequently confirmed by chief cytopathologist.

\section{Statistical analysis}

For categorical variables, summary tables will provide giving sample size, and relative frequencies .For continuous variables, a summary will provide the arithmetic mean and standard deviation (SD) .Quantitative data are shown as the mean \pm standard deviation, whereas numbers and percentage were provided for the qualitative data .Pearson's chi-squared was applied to test the correlations between the study groups for the categorical parameters . AP-value of $5 \%$ or less will be considered statistically significant .Statistical analyses were performed using SPSS version 25 (SPSS Inc., Chicago, IL, USA).

\section{Data collection:}

Patient files were reviewed for ND results (category I) to compare the two techniques. The following data were collected and analyzed: age, sex, anatomic location of the biopsy, thyroid nodule size, biopsy technique, TIRADS and FNA Bethesda results.

\section{Results}

A total of 357 patient files were reviewed; 290 (81\%) were females, and $67(19 \%)$ males with mean age of $56.9 \pm 13.5$ years. Demographic data of the two groups is presented in Table 1. No significant differences were found between the groups in terms of sex, age and nodule location and ACR-TIRADS scores (table 1 and table $2 \mathrm{a}$ and $\mathbf{2 b}$ ).

A total of 357 samples were obtained. 181 in the long axis approach and 176 in the short axis approach. The overall diagnostic adequacy of specimens obtained using the long axis approach, was significantly higher than that using the short axis approach ( $92.8 \%$ versus $83.3 \%$, respectively, $\mathrm{P}=0.006)$. 
Table 1: Demographic and clinical characteristics of all participants in the study according to the fineneedle aspiration (FNA) thyroid nodule biopsy technique (parallel vs. perpendicular).

\begin{tabular}{llll} 
Variables & $\begin{array}{l}\text { long Axis } \\
(n=181)\end{array}$ & $\begin{array}{c}\text { Short Axis } \\
(n=176)\end{array}$ & $p$ \\
\hline Gender, Female (n, \%) & $145,80.1$ & $145,82.4$ & 0.582 \\
\hline Age, years (mean $\pm S D)$ & $56.9 \pm 13.5$ & $57.1 \pm 15.2$ & 0.897 \\
\hline Side (n, \%) & & & \\
\hline Right & $90,49.7$ & $97,55.1$ & 0.595 \\
\hline Left & $83,45.9$ & $72,40.9$ & \\
\hline Isthmus & $8,4.4$ & $7,4.0$ & \\
\hline BETHESDA I (n, \%) & $13,7.2$ & $29,16.6$ & 0.006 \\
\hline TIRADS (n, \%) & & & \\
\hline 2-3 & $84,47.7$ & $76,50.0$ & 0.681 \\
\hline 4-5 & $92,52.3$ & $76,50.0$ & \\
\hline Diagnostic, Yes (n, \%) & $168,92.8$ & $145,83.3$ & 0.006
\end{tabular}

Table 2a: TIRADS levels for all nodules regardless of the approach $(n, \%)$.

\begin{tabular}{|c|c|c|c|}
\hline TIRADS & $2-3$ & 160 & 48.8 \\
\hline & $4-5$ & 168 & 51.2 \\
\hline
\end{tabular}

Table 2b: Correlation between BETHESDA I and TIRADS levels for all nodules regardless of the approach $(n, \%)$.

\begin{tabular}{lrll} 
& & $\begin{array}{l}\text { Nondiagnostic } \\
\text { Bethesda I } \\
(\mathrm{n}, \%)\end{array}$ & \\
& & & \\
\hline TIRADS & $2-3$ & $15,40.5$ & 0.287 \\
\hline & $4-5$ & $22,59.5$ & \\
\hline
\end{tabular}

Discussion 
US-guided FNAC is an accurate and effective tool in the workup of thyroid nodules. As mentioned, there are two accepted approaches for thyroid gland biopsy. In this study, we compared the adequacy of the two techniques. As hypothesized, our study showed that performing FNAC US guided using the long axis approach yields fewer ND (category I) results than the short axis $(7.2 \%$ vs $16.6 \%)$.

Although FNAC is a common procedure. Only one prior relatively small sample study has compared the short axis vs long axis approaches for FNAC in the thyroid gland. Kandil et al ${ }^{12}$ compared 49 long axis and 31 short axis FNAC samples. In their study, they found $95.1 \%$ of adequate samples in the long axis versus $93.9 \%$ in the short axis $(p<0.01)$. They also concluded that the long axis approach results in fewer ND (Category I) samples.

Although FNAC is considered an accurate and cost-effective method for the diagnostic evaluation of thyroid nodules, nondiagnostic results (Category I) are a prominent back draw of this procedure. The rate of ND (Category I) FNAC varies widely among studies, with rates as high as $47 \%$ and as low as $0.6 \% .{ }^{13-}$ ${ }^{14}$ Nondiagnostic results increase costs, patient stress, and time to diagnosis. Moreover, nondiagnostic samples can harbor a malignancy in $1-4 \%$ of cases. ${ }^{15}$

The superiority of the long axis technique can be explained by the fact that in this technique, the needle is navigated to the nodule under vision and not just by the operator experience in a blind way. Our results are compatible with other articles regarding US-guided nerve block that have also shown the superiority of the long axis technique for more accurate and efficient nerve block. Schafhalter-Zoppoth et al ${ }^{16}$ showed that the long axis is more accurate for achieving nerve block. In their study, there was significant improvement in achieving nerve block with this technique. Stone et al ${ }^{17}$, in their study regarding venous access ultrasound guidance, also stated that long access is associated with improved visibility of the needle tip during vessel puncture. They concluded that this approach helps decrease complications associated with ultrasound-guided central venous catheterization.

\section{Conclusion}

In summary, we found that the long axis approach for US-guided FNAC US guidance is independently more accurate and produces significantly fewer ND (category I) results than the short axis approach. This is the first large study comparing the two FNAC US-guided techniques regarding an accurate diagnosis.

Therefore, we suggest to prefer this technique for thyroid nodule FNAC.

\section{References}

1. Tamhane, S. \& Gharib, H. Thyroid nodule update on diagnosis and management. Clin Diabetes Endocrinol. 2016;2:17

2. Moon, J. H. et al. Prevalence of thyroid nodules and their associated clinical parameters: a largescale, multicenter-based health checkup study. The Korean Journal of Internal Medicine. 
2018;33(4):753-762.

3. Bestepe, N. et al. Is thyroid nodule volume predictive for malignancy? (Archives of Endocrinology and Metabolism, 2019).

4. Schenke, S. Diagnostic Performance of Different Thyroid Imaging Reporting and Data Systems (Kwak-TIRADS, EU-TIRADS and ACR TI-RADS) for Risk Stratification of Small Thyroid Nodules ( $\leq 10$ mm). Journal of Clinical Medicine, 9 (1), 236 (2020).

5. Mani, N. Investigating the value of fine needle aspiration cytology in thyroid cancer. Journal of Cytology, 28 (4), 185 (2011).

6. Lee, Y. B. et al. Current status and diagnostic values of the Bethesda system for reporting thyroid cytopathology in a papillary thyroid carcinoma-prevalent area.Head \& neck39,269-274(2017).

7. Cibas, E. S. Te 2017 Bethesda System for Reporting Tyroid Cytopathology. Tyroid: official journal of the American Thyroid Association, 27, 1341-1346 (2017).

8. Cristo, A. P. D. Increasing diagnostic effectiveness of thyroid nodule evaluation by implementation of cell block preparation in routine US-FNA analysis. Archives of Endocrinology and Metabolism, 60 (4), 367-373 (2016).

9. Williams, B. A. Rates of thyroid malignancy by FNA diagnostic category. J Otolaryngol Head Neck Surg, 42, 61 (2013).

10. Ycaza, A. E. E. D. et al. Risk of Malignancy in Thyroid Nodules with Non-Diagnostic Fine-Needle Aspiration: A Retrospective Cohort Study. Thyroid, 26 (11), 1598-1604 (2016).

11. Crockett, J. C. The thyroid nodule: fine-needle aspiration biopsy technique.J Ultrasound Med. 2011;30:685-694.

12. Kandil, E. et al. 12, "Comparison of Ultrasound-Guided Biopsy Technique for Thyroid Nodules with Respect to Adequacy of Cytological Material." Orl, vol. 73, no. 4, 2011, pp. 177-181.

13. Ceresini, G. et al. (2004) Ultrasound-Guided Fine-Needle Capillary Biopsy of Thyroid Nodules, Coupled with On-Site Cytologic Review, Improves Results. Thyroid 14 (5) 385-389.

14. Al Maqbali, T., Tedla, M., Weickert, M. O. \& Mehanna, H. 14,, : Malignancy risk analysis in patients with inadequate fine needle aspiration cytology (FNAC) of the thyroid. PLoS One 2012;7:e49078.

15. Cibas, E. S. \& Ali, S. Z. The Bethesda system for reporting thyroid cytopathology. Thyroid. 2009;19:1159-1165.

16. Schafhalterzoppoth, I. Ultrasound visibility of needles used for regional nerve block: An in vitro study. Regional Anesthesia and Pain Medicine, 29 (5), 480-488 (2004).

17. Stone, M. B. Needle tip visualization during ultrasound-guided vascular access: short-axis vs longaxis approach. The American Journal of Emergency Medicine, 28 (3), 343-347 (2010).

\section{Figures}




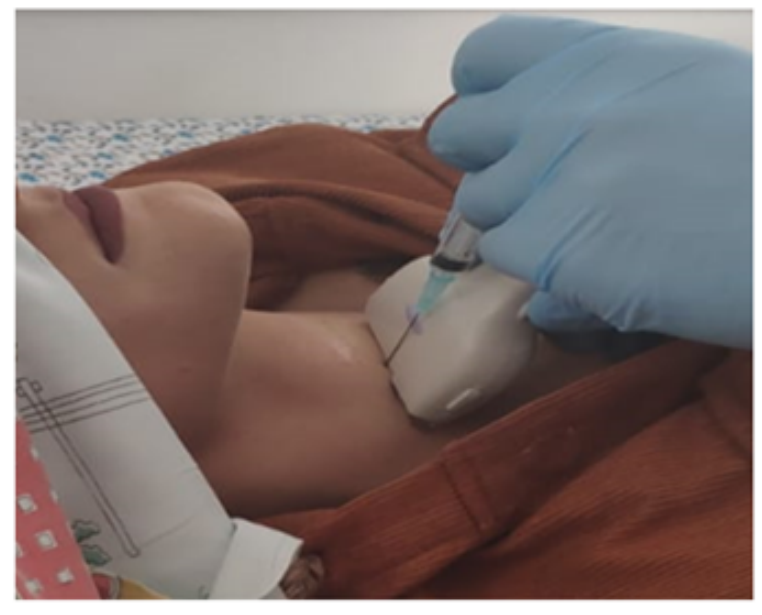

(Fig 1A-B).

PERPENDICULAR: Needle out of/parallel to transducer beam

Short axis approach, the ultrasound transducer is oriented transversely to the thyroid nodule (Fig 1A-B).With the short axis method, the path of the needle cannot be visualized. The tip of the needle is seen as a hyperechoic spot (Fig.1A-C)

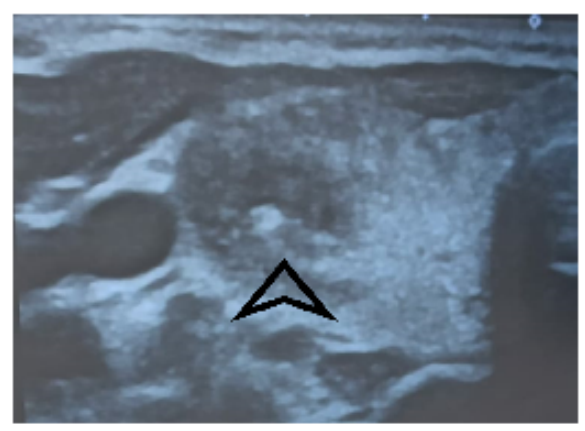

(Fig 1A-C)

\section{Figure 1}

"See image above for figure legend" 


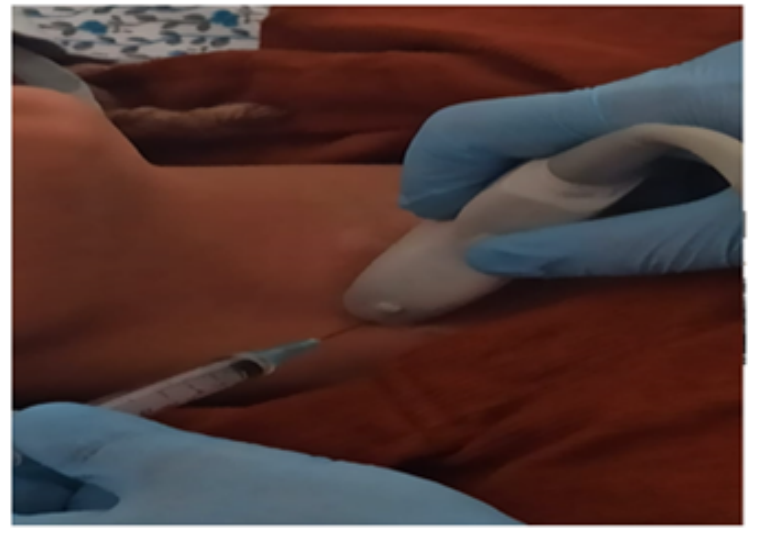

Long-axis technique with the needle lined up at the center of the short axis of the ultrasound probe as seen in Figure 2A-B. Long-axis technique allows for complete visualization of the needle from the dermis to thyroid nodule shown in Fig (Fig 2A-C)
(Fig 2A-B).

PARALLEL: Needle in/parallel to transducer beam

\section{Figure 2}

"See image above for figure legend" 